\title{
Computer Aided Reverse Engineering of a Toy Car
}

\section{Dr. Jahangir Ansari, Virginia State University}

Jahangir Ansari is Associate Professor of Manufacturing Engineering at Virginia State University. He received his M.S. degree in Mechanical Engineering in 1979 and Ph. D. degree in Mechanical Design and Production Engineering in 1983 both from Seoul National University. He joined the faculty at VSU in 2002. His research interests include Structural Vibration, FEM, CAD/CAM/CAE, and Virtual Manufacturing. 


\title{
Computer Aided Reverse Engineering of a Toy Car
}

\begin{abstract}
:
This paper focuses on a 3-D solid modeling technique employed in reverse engineering of a Toy Car. Engineering Graphics and CAD/CAM are two of the core courses taught in our Manufacturing Engineering program in sophomore and junior years respectively. The Engineering Graphics curriculum familiarizes students with 2-D drafting and 3-D solid modeling and assembly of simple products. In continuation, both computer aided design and manufacturing, as well as Rapid Prototyping application, are covered in the CAD/CAM course. Furthermore, application of computer aided technologies in manufacturing simulation and engineering analysis, as well as other areas of the students' interest, are practiced in under graduate research and/or individual studies. Earlier, a team of two students had been engaged in a project titled as: Computer Aided Reverse Engineering of a Toy Car. One of the main objectives of this project was for the students to extend their knowledge of reverse engineering and to also gain a hands-on experience in the field of solid modeling of complicated products. Coordinates Measurement Machine (CMM), a caliper, and a micrometer were used to measure the main dimensions of the Toy Car. A solid modeling program was then used for creating the model and manufacturing analysis. This paper reports the re-engineering methodology and process of the toy car model body as a manufacturing design project.
\end{abstract}

\section{Introduction:}

A survey conducted by Eggert ${ }^{1}$ indicates that both academia and industry are strongly in agreement that upon graduating, a mechanical engineering student should be capable of designing a part using CAD/Solid Modeling. Among the several competencies desired by the job market, engineers with these skills are highly in demand. Therefore, like most engineering programs ${ }^{2-4}$, our manufacturing engineering program at Virginia State University places a great emphasis on hands-on activities in part design and product assembly competencies in its curriculum.

Manufacturing engineering students develop skills for the various elements of the design process through the curriculum, culminating in a design implementation course during their senior year ${ }^{5}$. The program offers at least six courses in which engineering design practice is included. Computer aided design and drafting, as well as solid modeling of parts, are strongly emphasized in the Engineering Graphics curriculum. Integrated methodology of solid modeling, analysis, and rapid prototyping in a product design process are emphasized in the CAD/CAM course. Students are asked to search for a product consisting of two or more components and to use our facilities to design and build a prototype model. The projects are graded according to creativity, level of challenge, accuracy, and quality of final product (prototype model). Furthermore, application of computer aided technologies in manufacturing simulation and engineering analysis are practiced in under graduate research and/or individual projects. This paper reports the reverse engineering methodology and process of a toy car model as a manufacturing design project conducted by a team of two students as theirs undergraduate research efforts.

\section{Project selection:}

In this course the students were asked to search and find at least five products in which they were interested to work on as their manufacturing design project. The selected products should require the complete product design cycle including an integrated methodology of remodeling, analysis, rapid prototyping, and simulation or testing for manufacturability. The purpose of this activity was 
to help students "warm up" by generating a number of ideas before choosing a project. Among the five products such as a leisure boat propeller, a bearing puller, a stapler, a cell phone holder, and a toy car body; the last one was selected for the project. The car body selected for this project was originally made through the die casting process with the overall dimensions of 8” $\mathrm{L}$ x 3”W x 2" $\mathrm{H}$ as shown in Figure 1.

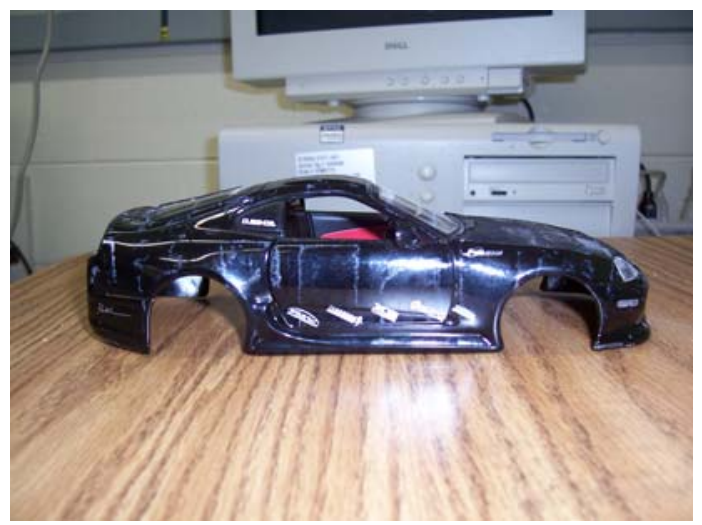

Fig. 1 - Selected toy car body.

\section{Geometry detection:}

The sequence for the reverse engineering of the product started with the geometric modeling utilizing a 3-D solid modeling software. In this process, students used their knowledge and skills of solid modeling and assembly, gained in the ENGR 200 course, and design and prototyping, learned at the beginning of the MANE 310 course, to design and visualize their selected ideas. Students realized that precise and exact indication of the geometry of the car body was quite a challenging process. A semi-automatic Coordinate Measurement Machine (CMM) was used to collect the coordinates of as many possible points on the surface to establish the profiles of the object on different planes. The CMM setup for identifying the points on the profiles is shown in Figure 2.

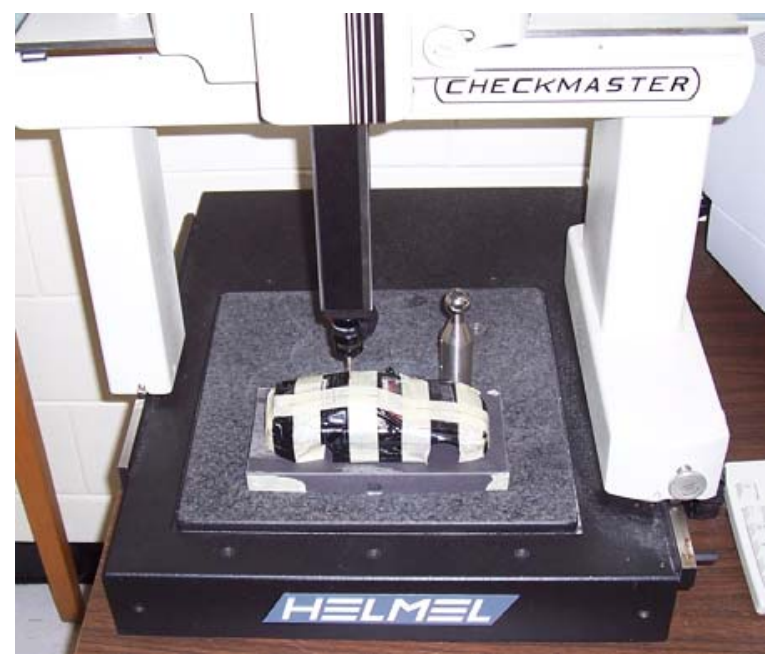

Fig. 2 - CMM setup and designated profiles. 
Some other challenges were faced using Matlab and Excel to manipulate the measured data for smoothness and symmetric characteristics of the car body profiles. Although creating a few numbers of profiles may not have caused serious difficulties for the students, creating many profiles to construct a complicated shape might require a specific procedure in order to accomplish the task in a shorter time. To minimize the risk of errors and for simplicity of any further modification, the application of macros in solid modeling process became very useful. The collected coordinates of sample points of some profiles before any manipulation are displayed in Figure 3.

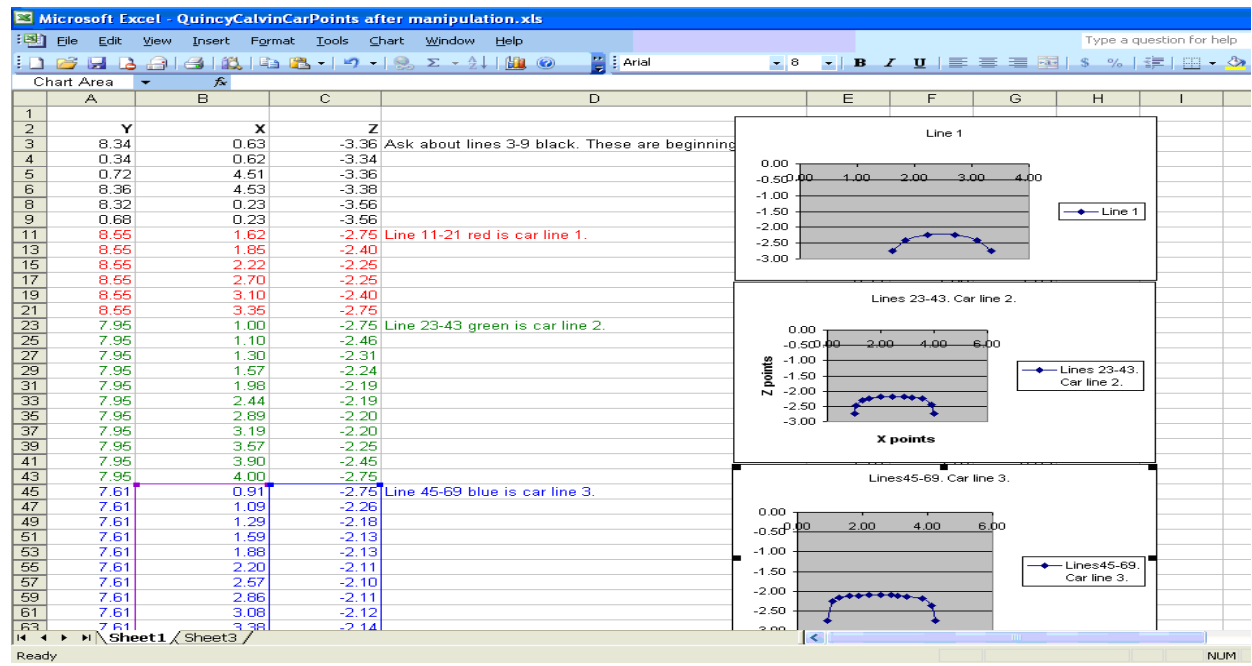

Fig. 3 - The collected coordinates of sample points in Excel

\section{Solid modeling process:}

After manipulating the raw data for smoothness and using the symmetric characteristics of the car body, a solid model of the car body was made using different tools in the SolidWorks software. For simplicity and further modifications and creating different shapes by changing the raw data, the macros application was highly beneficial. In this process, students used their knowledge and skills of solid modeling to design, visualize, modify, and change their selected product. The procedure of solid modeling the product is shown in Figure 4.
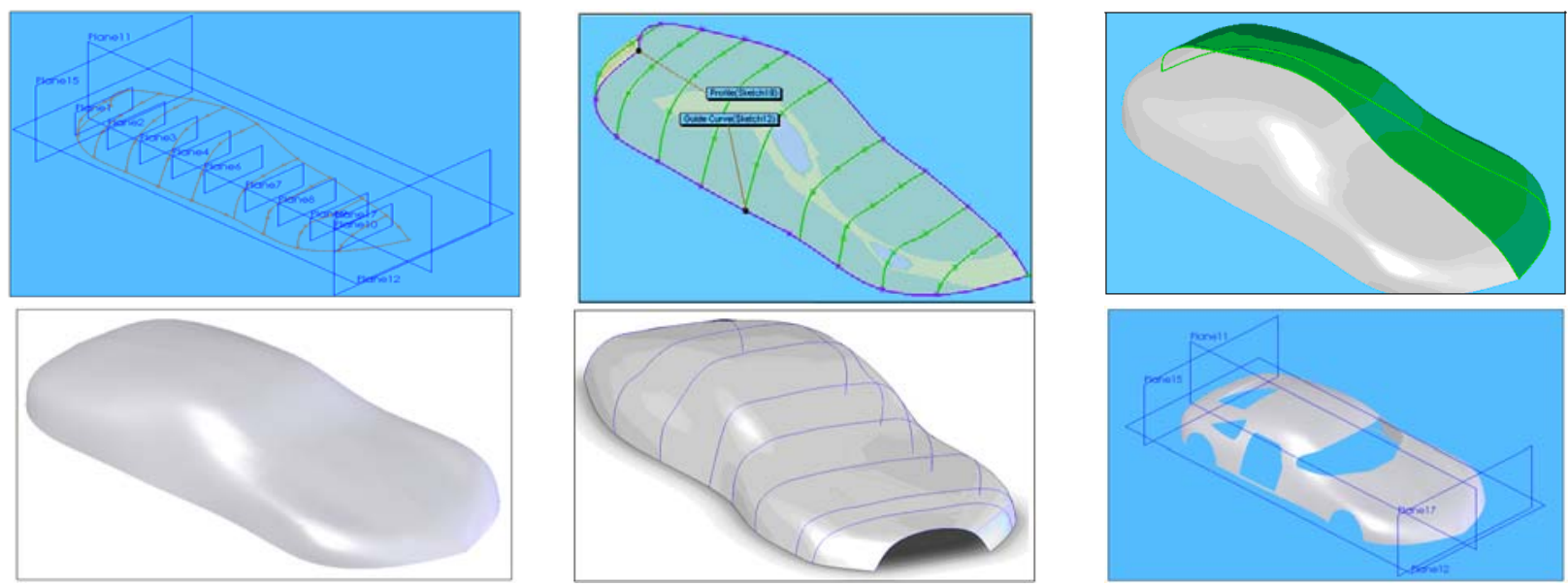

Fig. 4 - Procedure of 3-D solid modeling 
Having the manipulated coordinates of points constructing the profiles and macros in place, any modifications to create a new shape design was easily achievable. Figure 5 shows different models constructed by modifications of the original profiles.
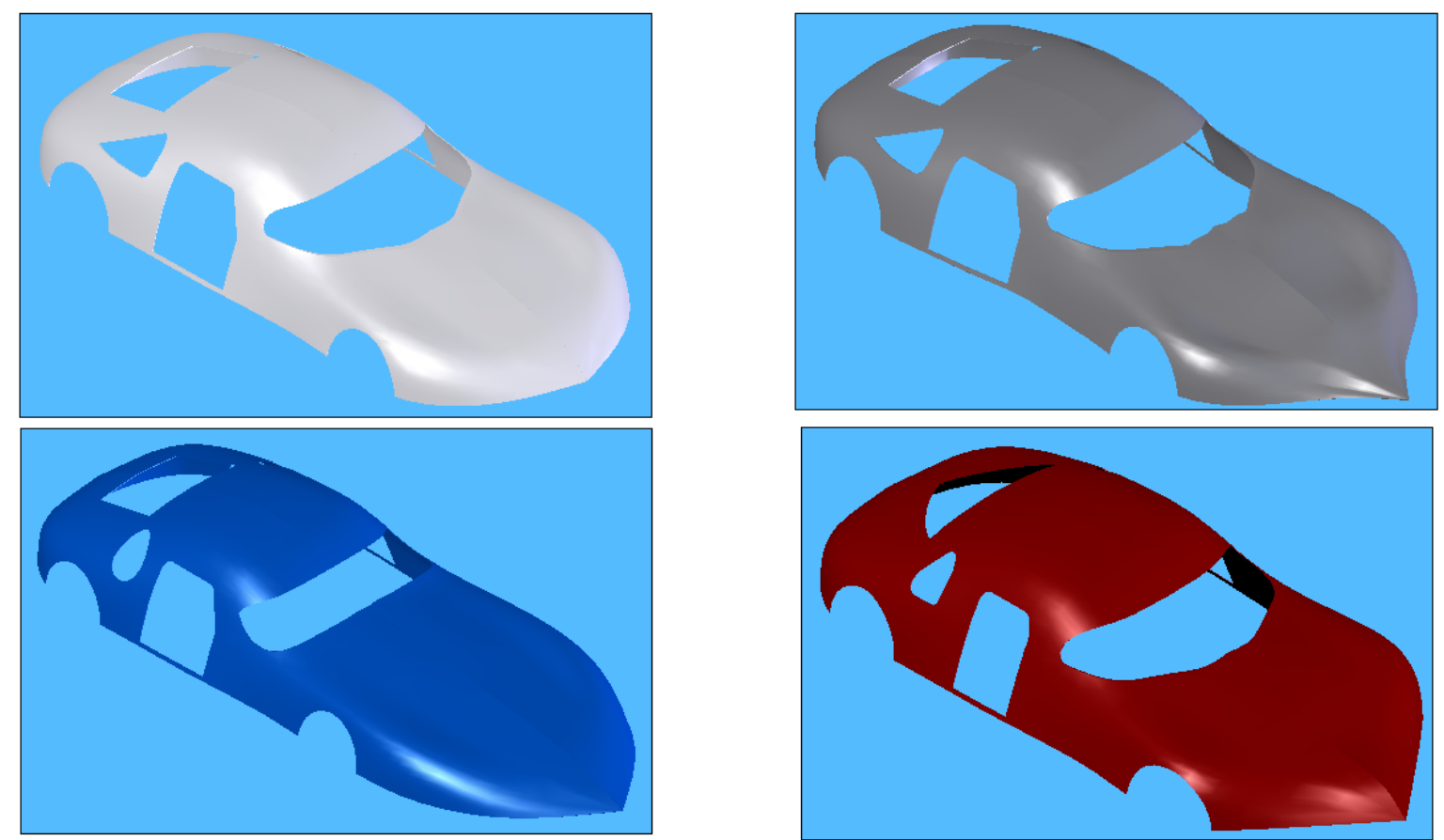

Fig. 5 - Modified 3-D models of the toy car body

\section{Stress Analysis of the product:}

To verify the validity of their design, students used Cosmos Express to figure out the critical points with high stress concentrations on the part. By changing the wall thickness and/or modifying the openings, they were easily able to find the more desirable options. They were also able to understand the concepts of loading, constraints, maximum deformation and many other mechanical and structural characteristics of their designed product. A sample of the stress distribution on the part simulated by Cosmos Express is show in Figure 6.
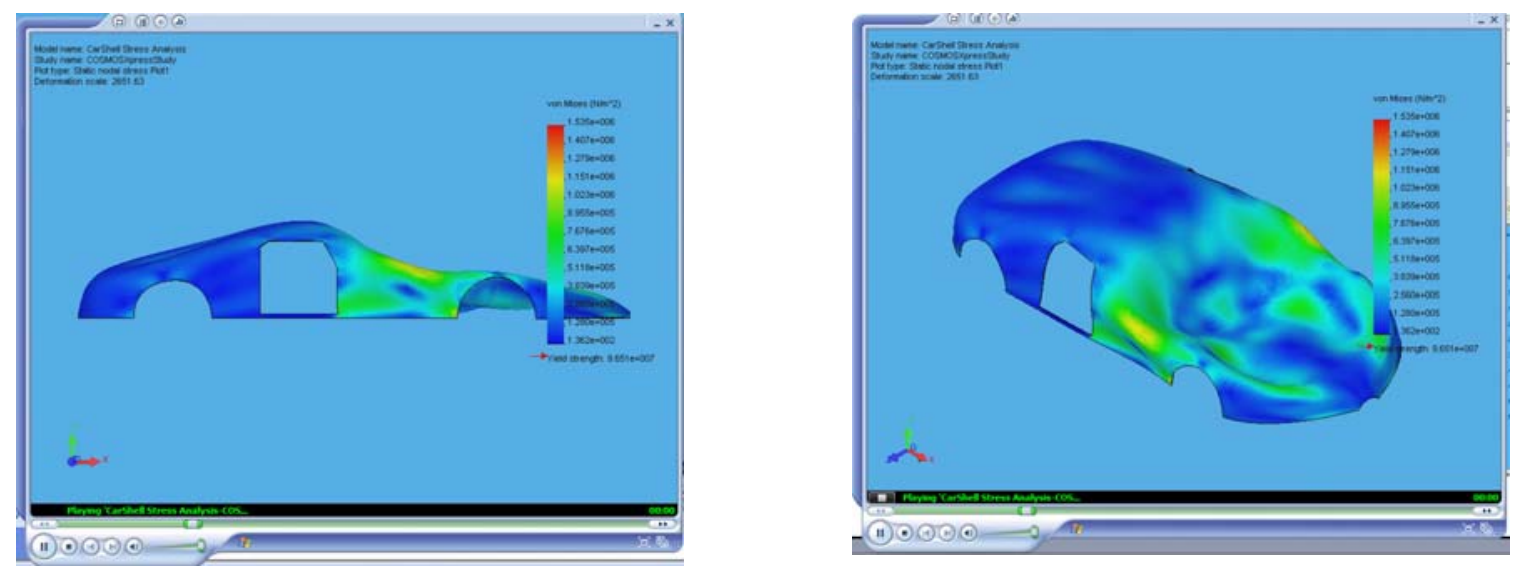

Fig. 6 - Simulated stress distribution on the car body 


\section{Material flow analysis:}

By selecting the material and manufacturing process suitable for the production, the students should be able to calculate the material and direct cost of the product. In this case, the toy car body could be produced by injecting plastic material into mold or using the aluminum die casting process. In either case, the Mold Flow Express was used to indicate the best injection points of the material as well as the manufacturing process of the part. Consequently the students were able to calculate the cost of the product. A sample of the material flow time simulated by Mold Flow Express is show in Figure 7.

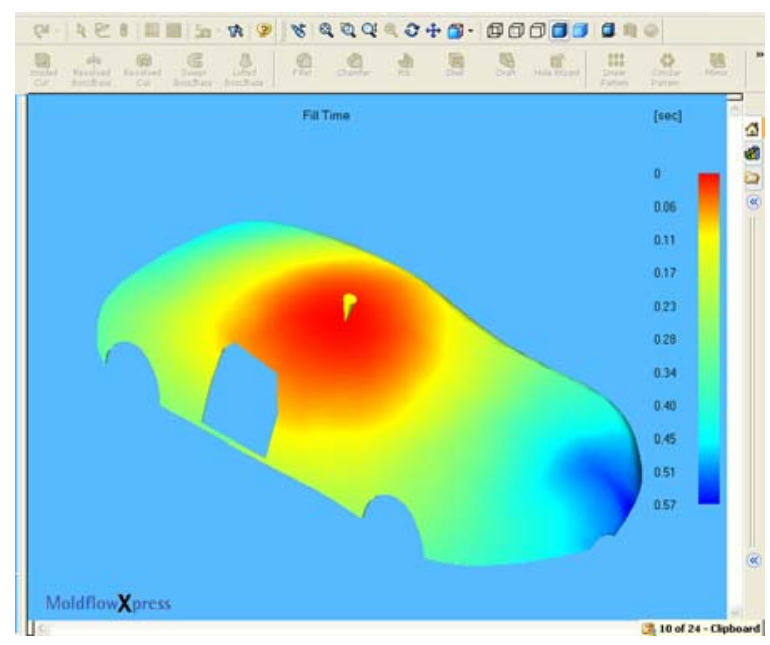

Fig. 7 - Simulated material flow time

Assessment:

The projects are evaluated according to creativity, level of challenge, accuracy, and quality of final deliverables. The selected projects are then presented to engineering faculty in Students' Projects Presentation day on the last week of each semester. This project was evaluated and gained recognition by winning the top award. The result of faculty evaluation is shown in Figure 8.

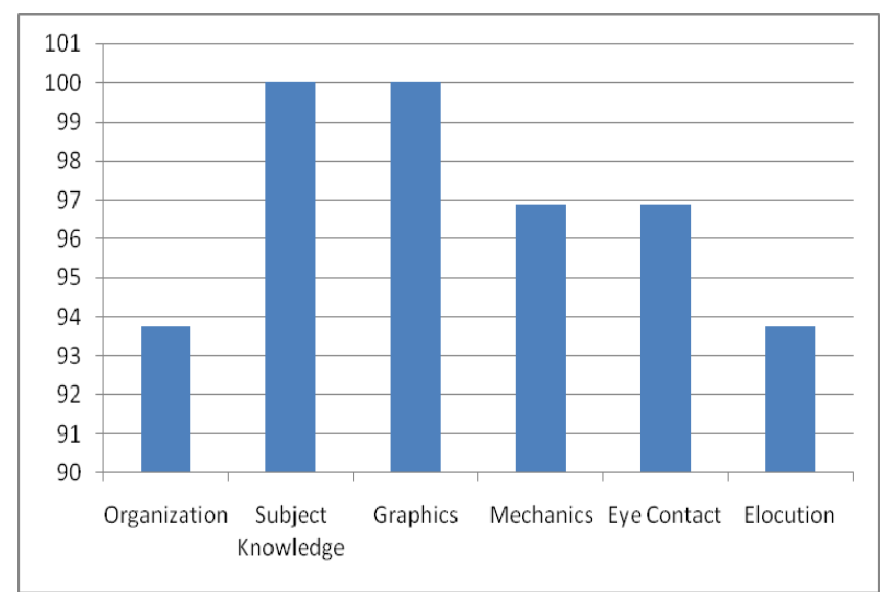

Fig. 8 - Project evaluation result 


\section{Conclusion:}

The reverse engineering project gave students an opportunity to develop their creativity, critical thinking, and hands-on skills in an area of their interest. In the project time frame, several short structured events were arranged, in which the students discussed the tasks that they had accomplished thus far, the challenges that they faced, and also their plans for completing their projects. This project helped the students to establish an understanding of how math and graphical tools in solid modeling process can be utilized to take maximum advantages in mechanical and manufacturing analyses of the models. The students also learned to understand the concepts of loading, constraints, maximum deformation and many other mechanical and structural characteristics of their designed product.

\section{Bibliography}

1. Rudolph Eggert, DESIGN FOR MANUFACTURE AND ASSEMBLY: A SURVEY OF DESIRED COMPETENCIES, Proceedings of the 2006 ASEE Annual Conference, Chicago, Illinois

2. Ronald E. barr, Philip S. Schmidt, Thomas J. Krueger, and Chu-Yun Twu, An Introduction to Engineering Through an Integrated Reverse Engineering and Design Graphics Project, Journal of Engineering Education, October 2000.

3. William Howard and Joseph Musto, Solid Modeling as the Cornerstone of an Introduction to Engineering Course, Proceedings of the 2006 ASEE Annual Conference, Chicago, Illinois.

4. R. Noorani, L. Rodriguez, M. Givens, and D. Christensen, PROJECT-BASED LEARNING IN A FRESHMAN COMPUTER GRAPHICS COURSE, Proceedings of the 2007 ASEE Annual Conference

5. Jahangir Ansari, Design Experience in a Manufacturing Engineering Program, Proceedings of the 2010 ASEE Annual Conference. 